UDC 615.1

DOI: $10.15587 / 2519-4852.2020 .210825$

\title{
REVIEW OF FORMULATION AND EVALUATION OF SELF-MICRO EMULSIFY- ING DRUG DELIVERY SYSTEM (SMEDDS)
}

\author{
Sanjib Bahadur, Kamesh Yadu, Pragya Baghel, Tripti Naurange, Manisha Sahu
}

Approximately half of the new drug applicants that reach formulation have poor water solubility. Oral delivery has been the main route of drug administration for the chronic treatment of numerous diseases. In different cases, in oral conveyance, $50 \%$ of the medication compound is hampered because of the high lipid soluble or fat soluble of the medication itself. Around $40 \%$ of new drug applicants show low solubility in water, which prompts poor oral bioavailability, high Intra and Intersubject changeability, and deficiency of dose proportionality

Aim of review. The main aim of this review article is to gather the information related to design and evaluation of SMEDDS. These information can be utilized to enhance the bioavailability of the poorly aqueous soluble drug for various types of orally administered drugs. In this review article, various literature are reviewed and summerised in single paper to serve as reference guide to various research scholars and researchers working on self-micro-emulsifying drug delivery systems.

Materials and Methods. To prepare this manuscript various keywords were searched in different search engine such as Google, Yahoo and Bing etc. This review article reviews the recent work done in the field of SMEDDS. It comprises review of literatures available in public domain and formulation of SMEDDS and its characterization is summarized in this article.

Result. The various strategies to developed poor aqueous soluble drug for improvement of bioavailability for example, salt development and molecular size reduction of the compound might be one technique to enhance the dissolution rate of the drug. In any case, these methods have their limitations. SMEDDS is one of the novel applications for the delivery of low water soluble and low bioavailability of drug. SMEDDS is a method to improve the aqueous solubility of the medication; SMEDDS are described as isotropic blenders of oils, surfactants, and co-surfactant. Upon slightly stir followed by dilution with distilled water, for example, gastrointestinal liquids, these techniques can define clear o/w micro emulsion. SMEDDS is first choice and key technology for developing the lipophilic drug and other different factors that chance to affect the oral bioavailability.

Conclusions. This review paper attempts to describe the preparation of SMEDDS and furthermore discusses the development of pseudo ternary phase diagram for SMEDDS. It describes the mechanism and method of preparation involved in SMEDDS. The capability of oral absorption of drug compound from the SMEDDS relies upon numerous formulation-related parameters, for example, surfactant concentration, oil/surfactant ratio, and hydrophobicity of emulsion, globule size and charge, in vitro, in vivo all of which basically characterized the ability of self-emulsification. SMEDDS are administered as unit dosage form and it also protect the degradation of drug.

Keywords: SMEDDS (self micro emulsifying drug delivery system), GI fluids (gastrointestinal fluids), o/w (oil in water), optimization, Isotropic mixture, X-ray powder diffraction (XPRD), zeta potential, surfactant, cosurfactant

Copyright (C) 2020, Sanjib Bahadur, Kamesh Yadu, Pragya Baghel, Tripti Naurange, Manisha Sahu. This is an open access article under the CC BY license (http://creativecommons.org/licenses/by/4.0).

\section{Introduction}

The various technique is utilized to enhance oral bioavailability of the poorly water-soluble drug, the oral route has been the ideal route of drug administration for the endless treatment of diseases as it offers more patient compliance [1]. $40 \%$ of new drug applicants show low solvency in water, which is a test being developed of the ideal oral solid dosage form as far as formulation development and bioavailability of new pharmaceutical preparation $[1,2]$. Various strategies are used for improving the bioavailability of those medications like the salt arrangement, $\mathrm{pH}$ change, $\beta$ cyclodextrin complex, microemulsion, and so forth [3]. SMEDDS is the best and most preferable method for the enhancement of oral bioavailability. SMEDDS are class of emulsion that has gotten specific consider- ation as a method for upgrading oral bioavailability of poor soluble drugs [4].

SMEDDS or self-emulsifying oil formulations (SEOF) are characterized as isotropic blends of natural and synthetic oils, delete these words, surfactants, or then again, at least one hydrophilic solvents and cosolvents/surfactants [2, 3]. SEDDS ordinarily produce emulsions with a globule size somewhere in the range of 100 and $300 \mathrm{~nm}$ while SMEDDS can have droplet size somewhere near $50 \mathrm{~nm}[3,5]$.

\section{Mechanism of self-emulsification}

The mechanism by which self-emulsification happens is not yet surely known. All things considered, it has been proposed that self-emulsification happens when the entropy change is the energy required to build the 
surface region of the dispersion to the higher than the preferring of dispersion.

The free energy of a simple emulsion preparation is a direct working of the energy required to make the most recent surface between the water and oil phases.
The two periods of the emulsion will intend to separate with time to decrease the interfacial region and in this manner the free energy of the system is done. Fig. 1 explain the mechanism of action of oral administration of SMEDDS on oral administration of drug [5].

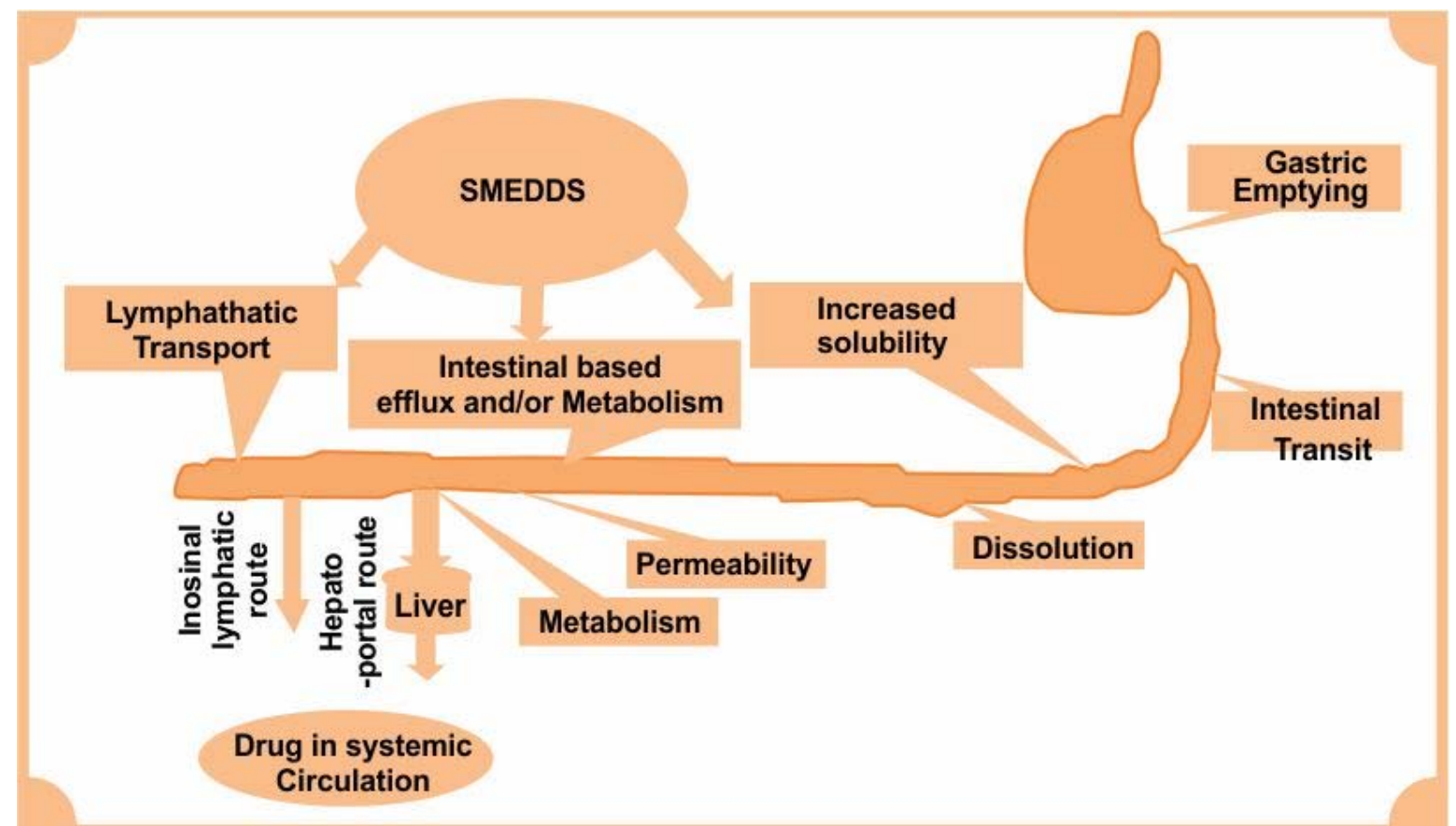

Fig.1. Mechanism of action of self-micro-emulsifying drug delivery system on oral administration of drug

\subsection{Advantages of SMEDDS}

Although SMEDDS formulation has several advantages:

1. Minimizing disturbance with the contact of GIT and gut wall.

2. Deliver peptides that are inclined to enzymatic hydrolysis in GIT.

3. It gives a sustained release of medicaments when a polymer is consolidated.

4. Safe and simple synthesis of SMEDDS.

5. More predictable fleeting profiles of medication assimilation.

6. Particular medication focusing on a particular replace ingestion with absorption window in the GI tract. the gut.

7. Drug security from the threatening condition in

8. The tale approach will upgrade water dissolvability and ultimate improve the availability of the lipophilic medication.

9. It demonstrates large inter and Intrasubject variable in absorption prompting variance over plasma profile solid or liquid dosage forms [6].

\subsection{Limitations of SMEDDS}

There are certain limitations associated with this system:

(1) Drug precipitation on dilution: diluted SMEDDS endure precipitation of drug into the gastrointestinal fluid. A regular need for the lipophilic formulations will be that they might have the capacity to have the drug in the solubilized manifestation in the gastrointestinal tract (GIT).
Precipitation of the drug from the system nullifies the benefits accessible by the lipid-based formulation system [7].

(2) The precipitation property of the formulation on dilution impact will be higher because of the impact of the hydrophilic solvents. It also requires the addition of polymers to decrease drug precipitation in vivo [7].

(3) Encapsulation in soft gelatine capsules: a large portion of the marketed SMEDDS preparations are accessible a soft gelatine capsule. Be that as it may, the gelatine capsule is related to few disadvantages. Assembling cost, transmissible spongiform encephalopathy (TSE), and purchaser inclination/religion are a couple of issues related to animal gelatine. Volatile co-solvents self-micro emulsifying drug delivery system are as well known to transmigrate into the shells of hard or soft gelatine capsule, ensure about the precipitation of the lipophilic medications. These issues drive the market necessity to locate a substitute for a soft gelatine capsule. The ongoing corresponding material of choice are those formulated from HPMC. The HPMC capsule shell has been investigated as a complementary methodology for encapsulating super saturable SMEDDS formulation [7].

(4) Storage and handling: liquid SMEDDS create issues in storage, stability, and handling. As such, SSMEDDS has all the earmarks of being an all sensible answer to address these issues [7].

(5) Limited targeting to lymphatic: focusing on lymphatics presents two essential advantages over conventional assimilation through the portal blood. To start with, transport through the intestinal lymph avoids presystemic hepatic metabolism and thereby improves the 
concentration of orally administered medications reaching the systemic circulation. Second, a site-specific drug administered to lymphatic organs could be accomplished. Normally high triglyceride solubility and high $\log \mathrm{P}$ is required for lymphatic transport. However, the amount of drug transported into lymphatics is variable from drug to drug. Thus, lipophilicity and triglyceride solvency of the drug in relationship with the lymphatic vehicle should be understood and a more satisfactory prescient model is needed. [7].

(6) Lack of good in vitro models: another difficulty in the improvement of self-micro emulsifying drug delivery systems and other lipid-based arrangements is the absence of good predictive in-vitro models for the assessment of the formulation. Traditional dissolution strategies do not work, as these preparations theoretically are reliant on the metabolism of lipid in the gut, preceding to release of the drug. However, to imitate this, an invitro model simulating the metabolism of the duodenum has been established. This in-vitro model needs further modification and validation before its strength can be evaluated. Further, improvement can be based on in-vitro in-vivo relationships, and hence various model lipidbased preparations should be established and assessed invivo in an appropriate animal model [7].

(7) Oxidation and polymorphism of the lipids used in formulating SEDDS/SMEDDS: lipid excipients containing unsaturated fats and its subordinates are to lipid oxidation. This requires consideration of lipid soluble antioxidant in preparation of capsules. Polymorphism related with thermo-softening up lipid excipients requires explicit procedure control in their application, to diminish polymorphic changes of the excipient matrix [7].

\subsection{Composition of SMEDS}

Various literature survey which are used to oil/ surfactant and co-surfactant physical mixture of the formulation of SMEDDS in different ratio. Most of the literatures used to surfactant and co-surfactant mixture (Smix) with oil in different ratio are used to prepare the SMEDDS preparation. Various components are used in SMEDDS formulation. As show in the table below.

Table 1

Classification of excipients used in formulation of SMEDDS on the basis of various literatures survey

\begin{tabular}{|c|c|c|c|c|}
\hline No. & Oil & Surfactant & Co-Surfactant & Reference \\
\hline 1. & Capmul MCM C8 & Cremophor EL & Transcutol HP & {$[8]$} \\
\hline 2. & castor oil & Tween-20 & Propylene glycol & {$[9]$} \\
\hline 3. & Triacetin & Triton-X100 & Carbitol & {$[10]$} \\
\hline 4. & Castor oil & Capmul MCM, Kolliphor EL & Kolliphor RH 40 & {$[11]$} \\
\hline 5. & Capryol 90 & gelucire 44/14 & Tween 80 & {$[12]$} \\
\hline 6. & Isopropyl myristate (IPM) & Tween 80 & propylene glycol & {$[2]$} \\
\hline
\end{tabular}

\section{ponents:}

SMEDDS preparation contain following com-

1. Oil

2. Surfactant

3. Co-surfactant

4. Consistency builder

5. Polymer

6. Other components

1. Oils: the oil refers to the most significant excipient in the SMEDDS formulation. Without a doubt, it will solubilize the important measure of the poor soluble dug [6]. Both medium-chain triglyceride (MCT) and long-chain triglyceride (LCT) oils with various degrees of immersion have been utilized in the design of SMEDDS [13]. For example, corn oil, olive oil, soybean oil, hydrolyzed corn oil, castor oil, sesame oil, soyabean oil isopropyl myristate $[13,14]$.

2. Surfactant: surfactant molecules may be classified based on the nature of the hydrophilic group within the molecule [13]. The surfactants are defined as four main categories as follows [6].

a. Anionic surfactants: the anionic surfactant is a water-loving group that conveys a negative charge [13]. For example, carboxyl $\left(\mathrm{RCOO}^{-}\right)$, sulphonate $\left(\mathrm{RSO}^{3-}\right)$ or sulfate $\left(\mathrm{ROSO}^{3-}\right)$. Potassium laurate, Sodium lauryl Sulphate (SLS) [6].

b. Cationic surfactants: the cationic surfactant is a hydrophilic component that passes on a positive charge. For example, quaternary ammonium halide [6] c. Zwitterionic surfactants (also called Ampholytic surfactants): the ampholytic surfactant contains both a positive charge (+ve) and a negative charge (-ve.) Such as sulfobetaine $[6,13]$.

d. Non-ionic surfactants: the non-ionic surfactant is a polar group they do not pass any type of charge yet get its water solubility from highly hydrophilic groups, such as polyoxyethylene or hydroxyl, for example, tween. The non-ionic surfactant SMEDDS formulation is used with high HLB value and there surfactant capability ranges are 30-60\% $\mathrm{w} / \mathrm{w}$ of the preparation to appearance a stable selfmicro emulsifying drug delivery system (SMEDDS). Surfactants contain a high HLB, and polar groups help the rapid formation of oil/water droplets just as quickly disperse of the preparation in the fluid media. Nonionic surfactants are amphiphilic on character and they can solubilize a respectably high amount of hydrophobic drugs $[6,13]$.

3. Co-solvents: co-surfactant is an organic solvent, for example, ethanol, propylene glycol, and polyethylene glycol (PEG) are suitable for oral conveyance and they accelerate the dissolution of huge amounts of either the water-loving or the lipid-soluble drug surfactants [13]. These solvents will even work as cosurfactants in microemulsion preparation. Then again, liquor and other co-surfactants have the burden that of dissipating into the shells of the delicately wrapped gelatine or hard gelatine case unmistakable $[15,16]$. 
4. Consistency builder: additional material can be added to adjust the consistency of the emulsion; for instance, stearic corrosive, cetyl liquor, tragacanth, and beeswax, etc. [6].

5. Polymers: inert polymer matrix denoting from $5-40 \%$ of ingredient comparative to the weight, which is not ionizable at physiological $\mathrm{pH}$, and being able of developing matrix. Examples are HPMC, ethyl cellulose, etc [6].

6. Other components: various constituents may be flavours, antioxidant agent and $\mathrm{pH}$ adjusters. To be sure, properties of lipid products, mostly those with unsaturated lipids express peroxide materialization with oxidation. Free radicals, for example, Peroxyl (ROO), Alkoxide (RO), and Hydroxyl $(\mathrm{OH})$ can harm the drug and produce toxicity. Lipid peroxides may too be designed because of auto-oxidation, which increases with the unsaturation level of the lipid molecule. Hydrolysis of the lipid high speed up because of the $\mathrm{pH}$ of the solution or from required processing energy, such as ultrasonic radiation. Lipophilic antioxidants (for example $\alpha$-tocopherol, propyl gallate, BHT) may in this manner be required to maintain the oily substance of the SMEDDS [6].

Formulation design: formulation of SMEDDS involves the following steps.

(1) Selection of active pharmaceutical ingredient (API) for self-micro-emulsifying drug delivery system (SMEDDS)

(2) Screening of surfactant for emulsifying ability.

(3) Choice of excipients for self-microemulsifying drug delivery system (SMEDDS).

(4) The solubility of a drug in oils, surfactant, and co-surfactant.

(5) Construction of pseudo ternary phase diagram.

(6) Preparation of self-micro-emulsifying drug delivery system (SMEDDS).

(7) Factor influencing self-micro-emulsifying drug delivery system.

(8) Characterization and evaluation of SMEDDS.

(1). Selection of active pharmaceutical ingredient for SMEDDS: it is most significant to know that the active pharmaceutical ingredient of choice can similarly have a considerable effect on the different features of SMEDDS, for example, phase behaviour and microemulsion particle size [1].

Different physicochemical characteristics of the API, for example, $\mathrm{pKa}, \log \mathrm{P}$, atomic structure and weight, presence of the ionizable group, and the amount have a significant effect on the functioning of SMEDDS [18]. Drugs that have low therapeutic dose are standard drug applicants for SMEDDS [19]. One of the major difficulties in developing oral preparation is keeping up active pharmaceutical ingredient soluble inside the G.I.T. furthermore, specifically, capitalize on drug dissolvability inside the major absorptive site of the gut drugs which are administered at extremely high dose are not appropriate for SMEDDS, except if they have great solubilization in at any rate one of the excipients of SMEDDS, ideally lipophilic phase.

The drug must be stable physically and chemically in the preparation and the drug discharge rate design must remain stable during the self-life of the SMEDDS [18].
(2). Screening of surfactant for emulsifying ability: the different surfactants are screened for their emulsification capacity. Surfactants can be added to the particular oil in 1:1 ratio. The admixture is homogenized. A fixed quantity of isotropic admixture is diluted with double purified water to yield a clear emulsion $[19,20]$. The resulting emulsions can be inspected outwardly for their relative polluting influence and their transmittance can be evaluated in UV-visible spectrophotometer with the help of double purified water as the blank [21-23].

(3). Selection of excipients: the excipients should be preferred from the record of generally regarded as safe "GRAS" excipients printed by USFDA [19, 24]. Good consideration of the physical appearance of excipients and their performance in preparation is the essential desire for effective preparation development [25]. In orderliness to prepare an effective SMEDDS for highest therapeutic outcomes, outstanding thought must be given to the following components;

- physicochemical characteristics of the API as well as excipients;

- development for drug excipients collaboration;

- physiological aspects that stimulate or restrain the bioavailability;

- biopharmaceutical features such as solubilization capacity, physical state regulatory status, miscibility, of the excipients at $25^{\circ} \mathrm{C}$;

- regulatory features of excipients;

- the temperature at which self-emulsification occurs.

When the preparation of SMEDDS, APIs must be consolidated into a suitable admixture of excipients; in this way, preparation enhancement usually begins with excipients assurance that was proposed to spare time and inexpensive. During the first choice of study, some excipients are recognized as potentially suitable for additional exploration attributable to their safety, sedate solvency, and their stability $[19,26]$.

(4). The solubility of a drug in oils, surfactant, and co-surfactant: the dissolvability of medication in oils, surfactant, and co-surfactant: the aggregate of the oils surfactants, and co-surfactants were screened for their attributes to dissolve a tremendous amount of pure drug [27]. An additional quantity of the drug is taken in clear screw cap glass vials that confine oil/surfactant/cosurfactant followed by blending on cyclomixer (vortex mixture). The admixture is shaken and centrifuged [28]. An aliquant part from the supernatant is withdrawn and further analyzed by UV-Visible Spectrophotometer at required $\mathrm{nm}[28,29]$.

(5). Construction of pseudo ternary phase diagram: different proportions of oil, surfactant, and cosurfactant are agitated to formulate various techniques $[30,31]$. Fixed quantity of each system is added in a beaker containing $0.1 \mathrm{~N} \mathrm{HCl}$ at $37^{\circ} \mathrm{C}$ and the substances are mixed using the magnetic stirrer $[32,33]$.

The clearness of the designed dispersion was visually examined with the help of following grading techniques;

A. Denoting the clear micro emulsion formation with bluish ting.

B. Denoting a translucent micro emulsion formation had a bluish appearance. 

tion.

C. Denoting a little less clear emulsion prepara-

D. Indicating a clear white emulsion development.

E. Signifying the details which had either poor emulsification with huge oil droplets superficially or the emulsion was not developed.

A phase diagram is developed to distinguish the results. Type A and B systems are most preferred because of the lower particle size. The following pyramid like structure over there shows the construction of pseudo ternary phase diagram. The following Fig. 2 demonstrate a sample of pseudo ternary phase diagram [28, 34, $35]$.

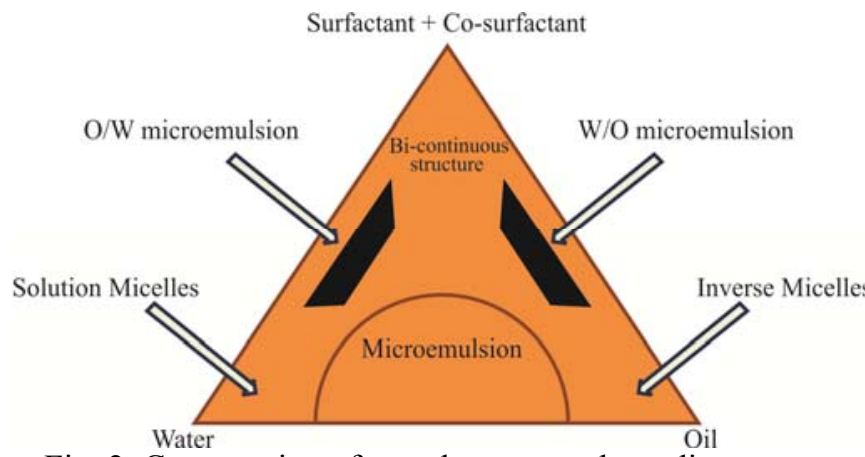

Fig. 2. Construction of pseudo ternary phase diagram

(6). Preparation of SMEDDS: the formulation contains the addition of the drug to the admixture of oil, surfactant, and co-surfactant and then it must be exposed to vertexing [36]. At that time, the drug is solubilized in any of the excipients and the remaining excipients are added to the drug solution $[37,38]$. At that point, the solution must be appropriately admixture and tested for the indication of impurity [39, 40]. After equilibration at atmospheric temperature for 48 hours, the solution must be heated for the development of a fine solution, whenever required [41, 42]. Contingent on the final volume, the preparation must be put away in capsules of appropriate size. The general technique of preparation a self- micro-emulsifying drug delivery system and their resulting form to micro emulsion /nano-emulsion is shown in Fig. $3[36,43]$.

\section{(7). Factor affecting SMEDDS:}

(a) API dose: generally drugs having a low therapeutic dose is preferred for the formulation of SMEDDS. However, such drugs are highly soluble in any constituents of SMEDDS specifically in the lipid phase. The drug which is not well dissolvable both in oil and water and has low Log P-value (around 2) is not an appropriate contender for SMEDDS [6].

(b) Drug solubility in the oil phase: the solubility of drugs in the oil phase influenced the capacity of the SMEDDS system in keeping the medication in the solution state. Right when the medication is solvent with the assistance of surfactant and co-surfactant the weakening of SMEDDS can bring about diminishing the dissolvable ability of surfactant, thereby coming about precipitation [6].

(c) The polarity of lipid phase: it is one of the variables that oversee the release of the micro emulsion. HLB, chain length and degree of unsaturation of the unsaturated fat, molecular weight of the water-loving segment and concentration of the emulsifier control polarity of the globules. Indeed, the polarity reproduces the affinity of the drug for oil as well as water and the kind of involved energies. The maximum polarity will improve the high degree of release of the drug into the aqueous phase. The maximum release was achieved with the preparation that had an oily state with maximum polarity.

The stability of dissolvability estimation can be completed to anticipate potential cases of precipitation in the gut. However, crystallization could be delayed insolubilizing and colloidal become stable out the condition of the gut. Studies express that such formulation can take up to 5 days to arrive at equilibrium and that medication can remain supersaturated state up to 24 hours after the primary emulsification occasion [6].

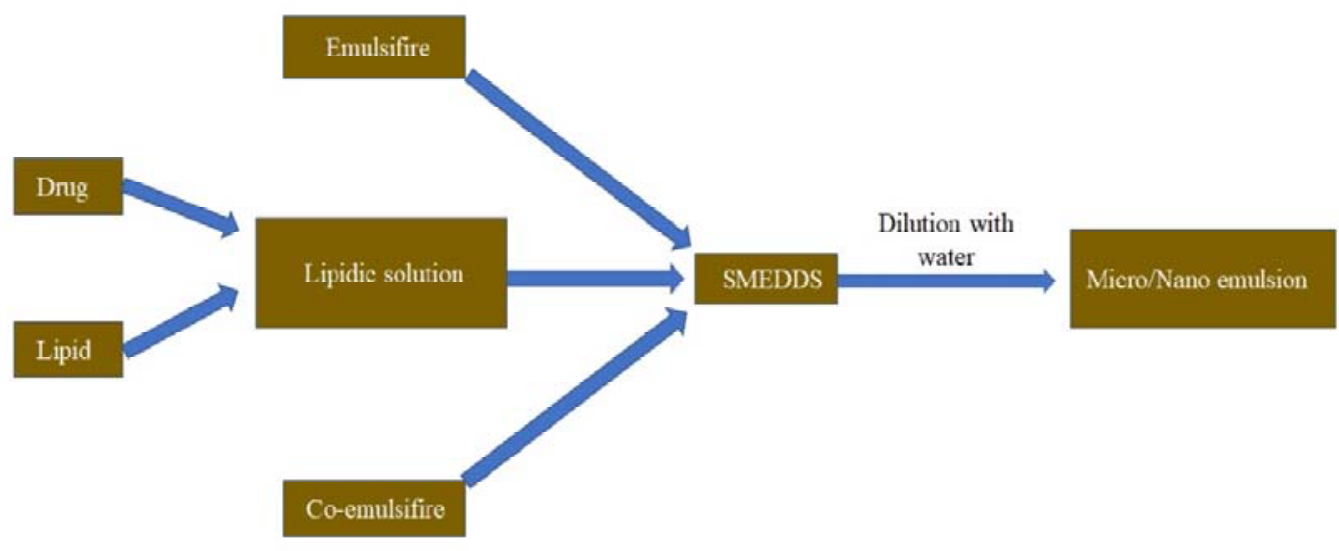

Fig.3. The general technique of preparation a self-micro-emulsifying drug delivery system and their resulting form to micro emulsion /nano-emulsion.

\section{SMEDDS:}

(8). Characterization and evaluation of

The efficiency of self-micro-emulsification could be estimated by determining the evaluation parameter [36].

1. Visual evaluation: the evaluation of selfemulsification is achieved by visual assessment. After dilution of SMEDDS with water, the impervious and milky white occurrence shows the development of macro emulsion though the fine, isotropic, clear solution shows the development of micro emulsion [36]. The preparation can be considered as constant when drug precipitation is not distinct [44]. Precipitation is normal if the preparation 
contains water soluble co-solvents and can be prevented by increasing the concentration of surfactant $[36,45]$.

2. Droplet size and particle size measurement: the globule size of the microemulsion is the measurement by PCS (photon correlation spectroscopy) or SEM (Scanning Electron Microscopy) which can evaluate sizes between 10-5000 $\mathrm{nm}[46,47]$. The nanometric size range of the molecule is held significantly after 100 or 1000 times diluted with distilled water, which identifies the system's compatibility with excess water [6]. Globule size is significant factors in SMEDDS because it impacts the rate and extent of drug discharge it also influences the stability of the micro-emulsion the globule size of the micro-emulsion is estimation by PCS (which estimate the varieties in light scattering due to Brownian movement of the particles) [48-50] which can measure size range between in the range of 10 and $5000 \mathrm{~nm}$. Light scattering is observed at room temperature at a 900 angle after outer standardization with spherical polystyrene droplets [51-53].

3. Refractive index and percent transmission: refractive index and percentage transmittance show the transparency of preparations $[54,55]$. The refractive index (RI) of the SMEDDS is a determined with refractometer and differentiates with water one [56]. The percent transmittance of the approach is determined at a specific wavelength (WL) using an ultraviolet-visible spectrophotometer maintaining purified water as blank if the refractive index (RI) of the system must be like that of water [57]. Preparation demonstrating transmittance $>99 \%$ is transmittance in nature $[58,59]$.

4. Zeta potential measurement: zeta potential is normally determined by the zeta potential analyzer or zeta meter system [60]. The estimation of the zeta potential analyzer indicates the constancy of emulsion after appropriate dilution [61]. Higher zeta potential shows a better constancy of the formulation. Generally, the value of zeta meter system is negative as a result of the free unsaturated fats yet when cationic lipid [62, 63]. For example, oleylamine is utilized, the charge contains the property of interfacing successfully with the mucosal surface of the GIT and these interchanges are electrostatic nature because of which solid adhesion can be estimated with improved absorption [36, 64].

5. Differential scanning colorimetry. This is generally used for the evaluation of micro-emulsions that are constructed by dilution of self-micro-emulsifying drug delivery system (SMEDDS) in the designation of peaks equivalent to water $[36,65]$. The peaks give data about the condition of water like a bound state or free state [66]. Purified water is utilized as a reference which shows high, sharp peaks around at $-170^{\circ} \mathrm{C}$ that demonstrates the point of freezing [67]. DSC experimentations microemulsions of water, surfactant and co-surfactant system and recognized identified peaks comparing to the water at a lower temperature than the purified water (roughly at $-450^{\circ} \mathrm{C}$ at $15 \% \mathrm{w} / \mathrm{w}$ ) demonstrating the appearance of water in the bound state in microemulsions ideally bound to surfactants $[68,69]$. A more high concentration of water than this prompts the move to a higher temperature [70]. From the examination of the thermal behaviour of water, they determined that the high concentration of water $(>35 \% \mathrm{~W} / \mathrm{W})$ manufactured $\mathrm{O} / \mathrm{W}$ micro emulsions $[36,71,72]$.
6. NMR techniques. The NMR technique after dilution of self-micro-emulsification systems to the prepared micro emulsion structure was determined. (FT-PGSE) Fourier transforms pulsed gradient spin-echo technique is used to examine the dispersive properties of microemulsion constituents. PGSE-NMR strategy is utilized to analyzed the progressions between micro emulsion phase and bi-consistent phase upon dilution with the help of 129xe NMR (nuclear magnetic resonance), the globule size of the micro emulsion can be measured with the help of observing the shift of signal to higher with particularly improve in the size of globules [36]. The self-dispersion NMR studies are utilized to evaluate the type of microemulsion that is formulated after dilution of SMEDDS and to evaluate the changes like $\mathrm{W} / \mathrm{O}$ (water in oil) to be continuous and bi-continuous to $\mathrm{O} / \mathrm{W}$ (oil in water) type upon increasing dilution. In this method, the self-diffusive coefficients of various constituents of micro-emulsion are differentiated with that of pure constituents. if the dispersion of one of the constituents is lower than that of pure constituents, this shows the existence of globules, that is, $\mathrm{O} / \mathrm{W}$ (oil in water) or W/O (water in oil), and generally, surfactant and co-surfactants as well have slow dispersion due to the development of layer around the globules by these constituents.

If the oil and water phase has high dispersion coefficients and is of a similar magnitude as pure constituents, it shows the presence of bi-continuous microemulsion [36].

7. Small angle $x$-ray and neutron scattering methods: this kind of technique is valuable for the estimation of structures that are formed by dilution of SMEDDS [72]. Assessment of fluid crystalline structures developed by the dilution of SMEDDS is significant as these control stability the preparation, self-emulsification, and amount of drug discharge X-Ray scattering examine on preparations containing various ratios of water. At $10 \% \mathrm{w} / \mathrm{w}$ (lower) water conc. lamellar structure was analyzed and at $20 \% \mathrm{w} / \mathrm{w}$ water concentration, lamellar structures were inspected [73]. Further increase in water concentration to $40 \% \mathrm{w} / \mathrm{w}$ uncovered hexagonal or lamellar structures. The temperature increasing to $37^{\circ} \mathrm{C}$ from $25^{\circ} \mathrm{C}$ did not cause important changes in fluid crystalline structures that are developed [74]. Small-angle neutron scattering techniques are helpful to examined transitions in micro-emulsion structures upon dilution and to observed the size and shape of the globules $[36,75]$

8. In vitro release. USP dissolution testing mechanical assembly type-II (paddle type) ought to be utilized for evaluation of the in-vitro drug discharge [76]. The dissolution test utilize a reasonable dissolution medium at $37^{\circ} \mathrm{C} \pm 5^{\circ} \mathrm{C}$. The rotation speed of the paddle should be maintained at $75 \mathrm{rpm}$ for $1 \mathrm{hrs}$. The study was performed as per the USP procedure. In dissolution vessel weight equivalent weight of preparation is added [77, 78]. At regular intervals, the appropriate volume of samples is withdrawn from the middle of vessels [79].

The sink condition is maintained by adding a fresh volume of dissolution media after withdrawing the sample $[80,81]$. After withdrawing the sample is filtered and observed in UV-spectrophotometer against blank in its respective range of $\lambda$ max. The percent of drug dissolved from preparation is determined. The above strategies were rehashed three times for every preparations $[28,82,83]$. 


\section{Stability}

(a) Temperature stability: by visual perception of the SMEDDS system at the different time span the shelf life as a function of time and the storage temperature is resolved [5]. By utilizing distilled water, preparations are diluted and are kept at a different scope of temperature (room temperature, $2-8^{\circ} \mathrm{C}$ or cooler) to check the temperature soundness of tests, and if any signs of phase separation, flocculation or precipitation is generally watched $[84,85]$.

(b) Centrifugation: to assess metastable system, the improved SMEDDS system are diluted with distilled water [5]. At this point, micro-emulsion are cen- trifuged at $1000 \mathrm{rpm}$ for 15 minutes at $0^{\circ} \mathrm{C}$ and inspected for any adjustment in the homogeneity of small scale emulsions [86, 87, 88].

10. In-vivo studies: there are the number of models that are utilized for conducting in vivo studies.

Every in vivo studies involving animal, to be granted permission by animal ethics committees and the researches are mandated to follow those guidelines [89, 90]. The study was controlled in three groups involving six animals in each group were divided.

Table 2 explain the various types of animal model and route of drug administration in SMEDDS [91, 92].

Different animal models utilized for in-vivo absorption studies in SMEDDS

\begin{tabular}{|c|c|c|c|}
\hline Animal model & Drug & Route of drug administration & References \\
\hline New Zealand male rabbits. & Nimodipine & Oral & {$[93]$} \\
\hline Male albino Rat. & Acyclovir & Oral & {$[94]$} \\
\hline Beagle dogs (Male). & Atorvastatin & Oral & {$[95]$} \\
\hline Female Wistar Rats. & Exemestane & Oral & {$[96]$} \\
\hline
\end{tabular}

\section{Bioavailability study in rat}

Male rodents are arbitrarily separated into two groups, in particular free standard drug solution and SMEDDS formulation containing drug [97]. Prior to the analysis, the rodents are housed for $72 \mathrm{~h}$ to adapt to the laboratory conditions and free access to water can be permitted. The two groups were given a similar dose [98]. Blood samples are collected at various time interval. The samples are centrifuged at $10,000 \mathrm{rpm}$ for 5-10 minute. The concentration of drug can be determined using HPLC [99-101].

\section{Conclusion}

Self-micro emulsifying drug delivery system formulation is formulated for the enhancement of oral bioavailability of poor soluble and low bioavailability of drug. It is most appropriate method to increase the drug solubility and bioavailability in oral administration of drug. The oil, surfactant and co-surfactant mixture is gently agitated followed by dilution of aqueous media. It forms clear oil/water micro emulsion. In this review, various components for preparation of SMEDDS is studied to help the researchers to choose the most appropriate component for their research. There are various types of evaluation parameter which are used to assess SMEDDS like particle size and there crystalline structure, charge of the particle in the formulation. This article also discusses various in vivo models to study characterize SMEDDS. This review article will serve as a base for the researchers working on SMEDDS, techniques used for enhancement of bioavailability, aqueous solubility of drugs.

\section{Conflicts of interest}

The authors declare that they have no conflicts of interest.

\section{References}

1. Maurya, S. D., Arya, R. K., Rajpal, G., Dhakar, R. C. (2017). Self-micro emulsifying drug delivery systems (smedds): a review on physico-chemical and biopharmaceutical aspects. Journal of Drug Delivery and Therapeutics, 7 (3), 55-65. doi: http://doi.org/10.22270/jddt.v7i3.1453

2. Ramya, A. R., Sudheer, P., Mohameid, A. S., Das, A. K. (2019). Design and Evaluation of a Self-Emulsifying Drug Delivery System of Aripiprazole. Indian Journal of Pharmaceutical Sciences, 81 (6), 1089-1098. doi: http://doi.org/10.36468/pharmaceuticalsciences. 607

3. Gumaste, S. G., Freire, B. O. S., Serajuddin, A. T. M. (2017). Development of solid SEDDS, VI: Effect of precoating of Neusilin ${ }^{\circledR}$ US2 with PVP on drug release from adsorbed self-emulsifying lipid-based formulations. European Journal of Pharmaceutical Sciences, 110, 124-133. doi: http://doi.org/10.1016/j.ejps.2017.02.022

4. Ramesh, B. J., Ramu, A., Vidyadhara, S., Balakrishna, T. (2019). Design and Evaluation of Telmisartan SMEDDS for Enhancing Solubility and Dissolution Rate. International Journal of Pharmaceutical Sciences and Nanotechnology, 12 (6), 47214730. doi: http://doi.org/10.37285/ijpsn.2019.12.6.8

5. Patel, M. J., Patel, S. S., Patel, N. M., Patel, M. M. (2010). A self-microemulsifying drug delivery system (SMEDDS). International Journal of Pharmaceutical Sciences Review and Research, 4 (3), 29-35. doi: http://doi.org/10.14843/jpstj.70.32

6. Kalamkar, P., Pawar, K., Baddi, H., Thawkar, B., Yevale, R., Kale, M. (2016). A Review on " Self Micro Emulsifying Drug Delivery System (SMEDDS ). Indian Journal of Drug, 4 (3), 361-373.

7. Dokania, S., Joshi, A. K. (2014). Self-microemulsifying drug delivery system (SMEDDS) - challenges and road ahead. Drug Delivery, 22 (6), 675-690. doi: http://doi.org/10.3109/10717544.2014.896058

8. Patel, M. H., Sawant, K. K. (2019). Self microemulsifying drug delivery system of lurasidone hydrochloride for enhanced oral bioavailability by lymphatic targeting: In vitro, Caco-2 cell line and in vivo evaluation. European Journal of Pharmaceutical Sciences, 138, 105027. doi: http://doi.org/10.1016/j.ejps.2019.105027

9. Aggarwal, G., Harikumar, S., Jaiswal, P., Singh, K. (2014). Development of self-microemulsifying drug delivery system and solid-self-microemulsifying drug delivery system of telmisartan. International Journal of Pharmaceutical Investigation, 4 (4), 195. doi: http://doi.org/10.4103/2230-973x.143123 
10. Shakeel, F., Raish, M., Anwer, M. K., Al-Shdefat, R. (2016). Self-nanoemulsifying drug delivery system of sinapic acid: In vitro and in vivo evaluation. Journal of Molecular Liquids, 224, 351-358. doi: http://doi.org/10.1016/j.molliq.2016.10.017

11. Bolko Seljak, K., Ilić, I. G., Gašperlin, M., Zvonar Pobirk, A. (2018). Self-microemulsifying tablets prepared by direct compression for improved resveratrol delivery. International Journal of Pharmaceutics, 548 (1), 263-275. doi: http://doi.org/10.1016/j.ijpharm.2018.06.065

12. Pandit, A., Kedar, A., Koyate, K. (2020). Hollow pessary loaded with lawsone via self-microemulsifying drug delivery system for vaginal candidiasis. Journal of Drug Delivery Science and Technology, 60, 101955. doi: http://doi.org/10.1016/j.jddst.2020.101955

13. Reddy, S., Katyayani, T., Navatha, A., Ramya, G. (2011). Review on self micro emulsifying drug delivery systems. International Journal of Research in Pharmaceutical Sciences, 2 (3), 382-392.

14. Akiladevi, D. N. M. (2017). Formulation And Development Of Self Emulsifying Drug Delivery System For Few Drugs. International Journal of Pharmacy and Pharmaceutical Sciences, 8 (0975-1491), 1-133.

15. Yetukuri, K. S. P. (2012). Yetukuri and Sudheer. Internation Journal of Pharmaceutical Science and Research, 3 (10), $3550-3558$

16. Talele, S. G., Gudsoorkar, V. R., Pharmacy, M. V. P. C. (2016). Novel Approaches for Solidification of Smedds. International Journal of pharma and bio sciences, 4, 90-101.

17. Patil, A. S., Mahajan, H. D., Wagh, R. D., Deore, B. L., Mali, B. J. (2016). Self-micro emulsifying drug delivery system (SMDDS): a novel approach for enhancement of bioavailability. Pharma Science Monitor, 5 (1), 133-143. Available at: http://www.pharmasm.com/download1.php?articleid=712\&download_file=20140320110931_20140123221316_10_amod.pdf

18. Shah Sanket, P., Shah Mansi, D. A. Y. (2012). Self- Micro Emulsifying Drug Delivery System: A Novel Approach For Enhancement Of Oral Bioavailability Of Poorly Soluable Drugs. American Journal of PharmTech Research, 2249-3387, $194-215$.

19. Shi, C.-H., Cai, S., Zhang, X., Tang, xiaojiao, Suo, H., Yang, li, Zhao, Y. (2014). Self-microemulsifying drug-delivery system for improved oral bioavailability of 20(S)-25-methoxyl-dammarane-3beta;, 12beta; 20-triol: preparation and evaluation. International Journal of Nanomedicine, 9, 913-920. doi: http://doi.org/10.2147/ijn.s56894

20. Chaudhari, K. S., Akamanchi, K. G. (2019). Novel bicephalous heterolipid based self-microemulsifying drug delivery system for solubility and bioavailability enhancement of efavirenz. International Journal of Pharmaceutics, 560, 205-218. doi: http://doi.org/10.1016/j.ijpharm.2019.01.065

21. Timur, S. S., Yöyen-Ermiş, D., Esendağl1, G., Yonat, S., Horzum, U., Esendağlı, G., Gürsoy, R. N. (2019). Efficacy of a novel LyP-1-containing self-microemulsifying drug delivery system (SMEDDS) for active targeting to breast cancer. European Journal of Pharmaceutics and Biopharmaceutics, 136, 138-146. doi: http://doi.org/10.1016/j.ejpb.2019.01.017

22. Huang, W., Su, H., Wen, L., Shao, A., Yang, F., Chen, G. (2018). Enhanced anticancer effect of Brucea javanica oil by solidified self-microemulsifying drug delivery system. Journal of Drug Delivery Science and Technology, 48, 266-273. doi: http://doi.org/10.1016/j.jddst.2018.10.001

23. Cirri, M., Roghi, A., Valleri, M., Mura, P. (2016). Development and characterization of fast-dissolving tablet formulations of glyburide based on solid self-microemulsifying systems. European Journal of Pharmaceutics and Biopharmaceutics, 104, 19-29. doi: http://doi.org/10.1016/j.ejpb.2016.04.008

24. Janković, J., Djekic, L., Dobričić, V., Primorac, M. (2016). Evaluation of critical formulation parameters in design and differentiation of self-microemulsifying drug delivery systems (SMEDDSs) for oral delivery of aciclovir. International Journal of Pharmaceutics, 497 (1-2), 301-311. doi: http://doi.org/10.1016/j.ijpharm.2015.11.011

25. Chintalapudi, R., Murthy, T. E. G. K., Lakshmi, Kr., Manohar, Gg. (2015). Formulation, optimization, and evaluation of self-emulsifying drug delivery systems of nevirapine. International Journal of Pharmaceutical Investigation, 5 (4), 205. doi: http://doi.org/10.4103/2230-973x.167676

26. Nardin, I., Köllner, S. (2019). Successful development of oral SEDDS: screening of excipients from the industrial point of view. Advanced Drug Delivery Reviews, 142, 128-140. doi: http://doi.org/10.1016/j.addr.2018.10.014

27. Truong, D. H., Tran, T. H., Ramasamy, T., Choi, J. Y., Lee, H. H., Moon, C. et. al. (2015). Development of Solid SelfEmulsifying Formulation for Improving the Oral Bioavailability of Erlotinib. AAPS PharmSciTech, 17 (2), 466-473. doi: http://doi.org/10.1208/s12249-015-0370-5

28. Parakh, D. R., Patil, M. P., Sonawane, S. S., Kshirsagar, S. J. (2016). Application of factorial design approach in development and evaluation of self microemulsifying drug delivery system (SMEDDS) of mebendazole. Journal of Pharmaceutical Investigation, 47 (6), 507-519. doi: http://doi.org/10.1007/s40005-016-0279-3

29. Sunazuka, Y., Ueda, K., Higashi, K., Tanaka, Y., Moribe, K. (2018). Combined effects of the drug distribution and mucus diffusion properties of self-microemulsifying drug delivery systems on the oral absorption of fenofibrate. International Journal of Pharmaceutics, 546 (1-2), 263-271. doi: http://doi.org/10.1016/j.ijpharm.2018.05.031

30. Chou, Y.-C., Li, S., Ho, C.-T., Pan, M.-H. (2020). Preparation and evaluation of self-microemulsifying delivery system containing 5-demethyltangeretin on inhibiting xenograft tumor growth in mice. International Journal of Pharmaceutics, $579,119134$. doi: http://doi.org/10.1016/j.ijpharm.2020.119134

31. Zheng, D., Lv, C., Sun, X., Wang, J., Zhao, Z. (2019). Preparation of a supersaturatable self-microemulsion as drug delivery system for ellagic acid and evaluation of its antioxidant activities. Journal of Drug Delivery Science and Technology, 53, 101209. doi: http://doi.org/10.1016/j.jddst.2019.101209

32. Jo, K., Kim, H., Khadka, P., Jang, T., Kim, S. J., Hwang, S.-H., Lee, J. (2020). Enhanced intestinal lymphatic absorption of saquinavir through supersaturated self-microemulsifying drug delivery systems. Asian Journal of Pharmaceutical Sciences, 15 (3), 336-346. doi: http://doi.org/10.1016/j.ajps.2018.11.009

33. Shete, H., Sable, S., Tidke, P., Selkar, N., Pawar, Y., Chakraborty, A. et. al. (2015). Mono-guanidine heterolipid based SMEDDS: A promising tool for cytosolic delivery of antineoplastics. Biomaterials, 57, $116-132 . \quad$ doi: http://doi.org/10.1016/j.biomaterials.2015.03.040

34. Pandey, V., Kohli, S. (2017). SMEDDS of pioglitazone: Formulation, in-vitro evaluation and stability studies. Future Journal of Pharmaceutical Sciences, 3 (1), 53-59. doi: http://doi.org/10.1016/j.fjps.2017.02.003

35. Wu, L., Qiao, Y., Wang, L., Guo, J., Wang, G., He, W. et. al. (2015). A Self-microemulsifying Drug Delivery System (SMEDDS) for a Novel Medicative Compound Against Depression: a Preparation and Bioavailability Study in Rats. AAPS PharmSciTech, 16 (5), 1051-1058. doi: http://doi.org/10.1208/s12249-014-0280-y

36. Akula, S., Gurram, A. K., Devireddy, S. R. (2014). Self-Microemulsifying Drug Delivery Systems: An Attractive Strategy for Enhanced Therapeutic Profile. International Scholarly Research Notices, 2014, 1-11. doi: http://doi.org/10.1155/2014/964051 
37. Djekic, L., Janković, J., Rašković, A., Primorac, M. (2018). Semisolid self-microemulsifying drug delivery systems (SMEDDSs): Effects on pharmacokinetics of acyclovir in rats. European Journal of Pharmaceutical Sciences, 121, 287-292. doi: http://doi.org/10.1016/j.ejps.2018.06.005

38. Gao, H., Wang, M., Sun, D., Sun, S., Sun, C., Liu, J., Guan, Q. (2017). Evaluation of the cytotoxicity and intestinal absorption of a self-emulsifying drug delivery system containing sodium taurocholate. European Journal of Pharmaceutical Sciences, 106, 212-219. doi: http://doi.org/10.1016/j.ejps.2017.06.005

39. Akram, J., Khan, J., Asbi, A., Budiasih, S. (2014). Formulation And Development Of Self Micro- Emulsifying Drug. World Journal of Pharmaceutical Research, 3 (7), 105-124.

40. Hintzen, F., Perera, G., Hauptstein, S., Müller, C., Laffleur, F., Bernkop-Schnürch, A. (2014). In vivo evaluation of an oral self-microemulsifying drug delivery system (SMEDDS) for leuprorelin. International Journal of Pharmaceutics, 472 (1-2), 20 26. doi: http://doi.org/10.1016/j.ijpharm.2014.05.047

41. Sprunk, A., Strachan, C. J., Graf, A. (2012). Rational formulation development and in vitro assessment of SMEDDS for oral delivery of poorly water soluble drugs. European Journal of Pharmaceutical Sciences, 46 (5), 508-515. doi: http://doi.org/10.1016/j.ejps.2012.04.001

42. Rajpoot, K., Tekade, M., Pandey, V., Nagaraja, S., Youngren-Ortiz, S. R., Tekade, R. K. (2020). Self-microemulsifying drug-delivery system: ongoing challenges and future ahead. Drug Delivery Systems. Elsevier Inc., $393-454$. doi: http://doi.org/10.1016/b978-0-12-814487-9.00009-0

43. Chen, X.-Q., Ziemba, T., Huang, C., Chang, M., Xu, C., Qiao, J. X. et. al. (2018). Oral Delivery of Highly Lipophilic, Poorly Water-Soluble Drugs: Self-Emulsifying Drug Delivery Systems to Improve Oral Absorption and Enable High-Dose Toxicology Studies of a Cholesteryl Ester Transfer Protein Inhibitor in Preclinical Species. Journal of Pharmaceutical Sciences, 107 (5), 1352-1360. doi: http://doi.org/10.1016/j.xphs.2018.01.003

44. Tung, N.-T., Tran, C.-S., Pham, T.-M.-H., Nguyen, H.-A., Nguyen, T.-L., Chi, S.-C. et. al. (2018). Development of solidified self-microemulsifying drug delivery systems containing 1-tetrahydropalmatine: Design of experiment approach and bioavailability comparison. International Journal of Pharmaceutics, 537 (1-2), 9-21. doi: http://doi.org/10.1016/j.ijpharm.2017.12.027

45. Wei, Y., Ye, X., Shang, X., Peng, X., Bao, Q., Liu, M. et. al. (2012). Enhanced oral bioavailability of silybin by a supersaturatable self-emulsifying drug delivery system (S-SEDDS). Colloids and Surfaces A: Physicochemical and Engineering Aspects, 396, 22-28. doi: http://doi.org/10.1016/j.colsurfa.2011.12.025

46. Cheng, G., Hu, R., Ye, L., Wang, B., Gui, Y., Gao, S. et. al. (2015). Preparation and In Vitro/In Vivo Evaluation of Puerarin Solid Self-Microemulsifying Drug Delivery System by Spherical Crystallization Technique. AAPS PharmSciTech, 17 (6), 1336-1346. doi: http://doi.org/10.1208/s12249-015-0469-8

47. Ujhelyi, Z., Kalantari, A., Vecsernyés, M., Róka, E., Fenyvesi, F., Póka, R. et. al. (2015). The Enhanced Inhibitory Effect of Different Antitumor Agents in Self-Microemulsifying Drug Delivery Systems on Human Cervical Cancer HeLa Cells. Molecules, 20 (7), 13226-13239. doi: http://doi.org/10.3390/molecules200713226

48. Yang, S., Gursoy, R. N., Lambert, G., Benita, S. (2004). Enhanced Oral Absorption of Paclitaxel in a Novel SelfMicroemulsifying Drug Delivery System with or Without Concomitant Use of P-Glycoprotein Inhibitors. Pharmaceutical Research, 21 (2), 261-270. doi: http://doi.org/10.1023/b:pham.0000016238.44452.f1

49. Aswar, M., Bhalekar, M., Trimukhe, A., Aswar, U. (2020). Self-microemulsifying drug delivery system (SMEDDS) of curcumin attenuates depression in olfactory bulbectomized rats. Heliyon, 6 (8), e04482. doi: http://doi.org/10.1016/j.heliyon.2020.e04482

50. Visetvichaporn, V., Kim, K.-H., Jung, K., Cho, Y.-S., Kim, D.-D. (2020). Formulation of self-microemulsifying drug delivery system (SMEDDS) by D-optimal mixture design to enhance the oral bioavailability of a new cathepsin K inhibitor (HL235). International Journal of Pharmaceutics, 573, 118772. doi: http://doi.org/10.1016/j.ijpharm.2019.118772

51. Gumaste, S. G., Serajuddin, A. T. M. (2017). Development of solid SEDDS, VII: Effect of pore size of silica on drug release from adsorbed self-emulsifying lipid-based formulations. European Journal of Pharmaceutical Sciences, 110, 134-147. doi: http://doi.org/10.1016/j.ejps.2017.05.014

52. Prachi, S., Prajapati, S. K. K. S. U., Shipra, S., Ali, A. (2012). a Review on Self Micro Emulsifying Drug Delivery System: an Approach To Enhance the Oral Bioavailability of Poorly Water Soluble Drugs. International Research Journal of Pharmacy, 3 (9), 1-6.

53. Sha, X., Wu, J., Chen, Y., Fang, X. (2012). Self-microemulsifying drug-delivery system for improved oral bioavailability of probucol: Preparation and evaluation. International Journal of Nanomedicine, 7, 705-712. doi: http://doi.org/10.2147/ijn.s28052

54. Joyce, P., Dening, T. J., Meola, T. R., Schultz, H. B., Holm, R., Thomas, N., Prestidge, C. A. (2019). Solidification to improve the biopharmaceutical performance of SEDDS: Opportunities and challenges. Advanced Drug Delivery Reviews, 142, 102117. doi: http://doi.org/10.1016/j.addr.2018.11.006

55. Goyal, U., Gupta, A., Rana, A. C., Aggarwal, G. (2012). Self microemulsifying drug delivery system: A method for enhancement of bioavailability. International Journal of Pharmaceutical Sciences, 3 (1), 66-79.

56. Milović, M., Djuriš, J., Djekić, L., Vasiljević, D., Ibrić, S. (2012). Characterization and evaluation of solid selfmicroemulsifying drug delivery systems with porous carriers as systems for improved carbamazepine release. International Journal of Pharmaceutics, 436 (1-2), 58-65. doi: http://doi.org/10.1016/j.ijpharm.2012.06.032

57. Hyma, P., Abbulu, K. (2013). Formulation and characterisation of self-microemulsifying drug delivery system of pioglitazone. Biomedicine \& Preventive Nutrition, 3 (4), 345-350. doi: http://doi.org/10.1016/j.bionut.2013.09.005

58. Chen, X.-Q., Ziemba, T., Huang, C., Chang, M., Xu, C., Qiao, J. X. et. al. (2018). Oral Delivery of Highly Lipophilic, Poorly Water-Soluble Drugs: Self-Emulsifying Drug Delivery Systems to Improve Oral Absorption and Enable High-Dose Toxicology Studies of a Cholesteryl Ester Transfer Protein Inhibitor in Preclinical Species. Journal of Pharmaceutical Sciences, 107 (5), 1352-1360. doi: http://doi.org/10.1016/j.xphs.2018.01.003

59. Shahnaz, G., Hartl, M., Barthelmes, J., Leithner, K., Sarti, F., Hintzen, F. et. al. (2011). Uptake of phenothiazines by the harvested chylomicrons ex vivo model: Influence of self-nanoemulsifying formulation design. European Journal of Pharmaceutics and Biopharmaceutics, 79 (1), 171-180. doi: http://doi.org/10.1016/j.ejpb.2011.01.025

60. McConville, C., Friend, D. (2013). Development and characterisation of a self-microemulsifying drug delivery systems (SMEDDSs) for the vaginal administration of the antiretroviral UC-781. European Journal of Pharmaceutics and Biopharmaceutics, 83 (3), 322-329. doi: http://doi.org/10.1016/j.ejpb.2012.10.007

61. Qureshi, M. J., Mallikarjun, C., Kian, W. G. (2015). Enhancement of solubility and therapeutic potential of poorly soluble lovastatin by SMEDDS formulation adsorbed on directly compressed spray dried magnesium aluminometasilicate liquid loadable 
tablets: A study in diet induced hyperlipidemic rabbits. Asian Journal of Pharmaceutical Sciences, 10 (1), 40-56. doi: http://doi.org/10.1016/j.ajps.2014.08.003

62. Gu, M., Gong, M., Qian, Y., Yan, G. (2013). Development of a self-microemulsifying drug delivery system to enhance oral bioavailability of $\beta$-elemene in rats. Journal of Drug Delivery Science and Technology, 23 (5), 485-491. doi: $\mathrm{http}: / /$ doi.org/10.1016/s1773-2247(13)50070-0

63. Niederquell, A., Völker, A. C., Kuentz, M. (2012). Introduction of diffusing wave spectroscopy to study self-emulsifying drug delivery systems with respect to liquid filling of capsules. International Journal of Pharmaceutics, 426 (1-2), 144-152. doi: http://doi.org/10.1016/j.ijpharm.2012.01.042

64. Abdulkarim, M., Sharma, P. K., Gumbleton, M. (2019). Self-emulsifying drug delivery system: Mucus permeation and innovative quantification technologies. Advanced Drug Delivery Reviews, 142, 62-74. doi: http://doi.org/10.1016/j.addr.2019.04.001

65. Djekic, L., Jankovic, J., Čalija, B., Primorac, M. (2017). Development of semisolid self-microemulsifying drug delivery systems (SMEDDSs) filled in hard capsules for oral delivery of aciclovir. International Journal of Pharmaceutics, 528 (1-2), $372-$ 380. doi: http://doi.org/10.1016/j.ijpharm.2017.06.028

66. Cerpnjak, K., Pobirk, A. Z., Vrečer, F., Gašperlin, M. (2015). Tablets and minitablets prepared from spray-dried SMEDDS containing naproxen. International Journal of Pharmaceutics, 495 (1), 336-346. doi: http://doi.org/10.1016/j.ijpharm.2015.08.099

67. Li, Q., Zhai, W., Jiang, Q., Huang, R., Liu, L., Dai, J. et. al. (2015). Curcumin-piperine mixtures in self-microemulsifying drug delivery system for ulcerative colitis therapy. International Journal of Pharmaceutics, 490 (1-2), 22-31. doi: http://doi.org/10.1016/ j.ijpharm.2015.05.008

68. Krstić, M., Popović, M., Dobričić, V., Ibrić, S. (2015). Influence of Solid Drug Delivery System Formulation on Poorly WaterSoluble Drug Dissolution and Permeability. Molecules, 20 (8), 14684-14698. doi: http://doi.org/10.3390/molecules200814684

69. Shanmugam, S., Im, H. T., Sohn, Y. T., Kim, Y.-I., Park, J.-H., Park, E.-S., Woo, J. S. (2015). Enhanced oral bioavailability of paclitaxel by solid dispersion granulation. Drug Development and Industrial Pharmacy, 41 (11), 1864-1876. doi: http://doi.org/10.3109/03639045.2015.1018275

70. Kanwal, T., Kawish, M., Maharjan, R., Ghaffar, I., Ali, H. S., Imran, M. et. al. (2019). Design and development of permeation enhancer containing self-nanoemulsifying drug delivery system (SNEDDS) for ceftriaxone sodium improved oral pharmacokinetics. Journal of Molecular Liquids, 289, 111098. doi: http://doi.org/10.1016/j.molliq.2019.111098

71. Kumar, R., Khursheed, R., Kumar, R., Awasthi, A., Sharma, N., Khurana, S. et. al. (2019). Self-nanoemulsifying drug delivery system of fisetin: Formulation, optimization, characterization and cytotoxicity assessment. Journal of Drug Delivery Science and Technology, 54, 101252. doi: http://doi.org/10.1016/j.jddst.2019.101252

72. Čerpnjak, K., Zvonar, A., Vrečer, F., Gašperlin, M. (2015). Characterization of naproxen-loaded solid SMEDDSs prepared by spray drying: The effect of the polysaccharide carrier and naproxen concentration. International Journal of Pharmaceutics, 485 (1-2), 215-228. doi: http://doi.org/10.1016/j.ijpharm.2015.03.015

73. Garg, V., Kaur, P., Singh, S. K., Kumar, B., Bawa, P., Gulati, M., Yadav, A. K. (2017). Solid self-nanoemulsifying drug delivery systems for oral delivery of polypeptide-k: Formulation, optimization, in-vitro and in-vivo antidiabetic evaluation. European Journal of Pharmaceutical Sciences, 109, 297-315. doi: http://doi.org/10.1016/j.ejps.2017.08.022

74. Gupta, S., Chavhan, S., Sawant, K. K. (2011). Self-nanoemulsifying drug delivery system for adefovir dipivoxil: Design, characterization, in vitro and ex vivo evaluation. Colloids and Surfaces A: Physicochemical and Engineering Aspects, 392 (1), $145-$ 155. doi: http://doi.org/10.1016/j.colsurfa.2011.09.048

75. Kumar, B., Garg, V., Singh, S., Pandey, N. K., Bhatia, A., Prakash, T. et. al. (2018). Impact of spray drying over conventional surface adsorption technique for improvement in micromeritic and biopharmaceutical characteristics of selfnanoemulsifying powder loaded with two lipophilic as well as gastrointestinal labile drugs. Powder Technology, 326, 425-442. doi: http://doi.org/10.1016/j.powtec.2017.12.005

76. Silva, L. A. D., Almeida, S. L., Alonso, E. C. P., Rocha, P. B. R., Martins, F. T., Freitas, L. A. P. et. al. (2018). Preparation of a solid self-microemulsifying drug delivery system by hot-melt extrusion. International Journal of Pharmaceutics, 541 (1-2), 1-10. doi: http://doi.org/10.1016/j.ijpharm.2018.02.020

77. Parmar, N., Singla, N., Amin, S., Kohli, K. (2011). Study of cosurfactant effect on nanoemulsifying area and development of lercanidipine loaded (SNEDDS) self nanoemulsifying drug delivery system. Colloids and Surfaces B: Biointerfaces, 86 (2), 327-338. doi: http://doi.org/10.1016/j.colsurfb.2011.04.016

78. Inugala, S., Eedara, B. B., Sunkavalli, S., Dhurke, R., Kandadi, P., Jukanti, R., Bandari, S. (2015). Solid selfnanoemulsifying drug delivery system (S-SNEDDS) of darunavir for improved dissolution and oral bioavailability: In vitro and in vivo evaluation. European Journal of Pharmaceutical Sciences, 74, 1-10. doi: http://doi.org/10.1016/j.ejps.2015.03.024

79. Shakeel, F., Raish, M., Anwer, M. K., Al-Shdefat, R. (2016). Self-nanoemulsifying drug delivery system of sinapic acid: In vitro and in vivo evaluation. Journal of Molecular Liquids, 224, 351-358. doi: http://doi.org/10.1016/j.molliq.2016.10.017

80. Kuentz, M. (2011). Oral self-emulsifying drug delivery systems, from biopharmaceutical to technical formulation aspects. Journal of Drug Delivery Science and Technology, 21 (1), 17-26. doi: http://doi.org/10.1016/s1773-2247(11)50002-4

81. Kalam, M. A., Raish, M., Ahmed, A., Alkharfy, K. M., Mohsin, K., Alshamsan, A. et. al. (2017). Oral bioavailability enhancement and hepatoprotective effects of thymoquinone by self-nanoemulsifying drug delivery system. Materials Science and Engineering: C, 76, 319-329. doi: http://doi.org/10.1016/j.msec.2017.03.088

82. Aloisio, C., Bueno, M. S., Ponte, M. P., Paredes, A., Palma, S. D., Longhi, M. (2019). Development of solid selfemulsifying drug delivery systems (SEDDS) to improve the solubility of resveratrol. Therapeutic Delivery, 10 (10), 626-641. doi: http://doi.org/10.4155/tde-2019-0054

83. Balata, G., Eassa, E., Shamrool, H., Zidan, S., Abdo Rehab, M. (2016). Self-emulsifying drug delivery systems as a tool to improve solubility and bioavailability of resveratrol. Drug Design, Development and Therapy, 10, 117-128. doi: http://doi.org/10.2147/ dddt.s95905

84. Dhumal, D. M., Akamanchi, K. G. (2018). Self-microemulsifying drug delivery system for camptothecin using new bicephalous heterolipid with tertiary-amine as branching element. International Journal of Pharmaceutics, 541 (1-2), 48-55. doi: http://doi.org/10.1016/j.ijpharm.2018.02.030

85. Mekjaruskul, C., Yang, Y.-T., Leed, M. G. D., Sadgrove, M. P., Jay, M., Sripanidkulchai, B. (2013). Novel formulation strategies for enhancing oral delivery of methoxyflavones in Kaempferia parviflora by SMEDDS or complexation with 2-hydroxypropyl- $\beta$ cyclodextrin. International Journal of Pharmaceutics, 445 (1-2), 1-11. doi: http://doi.org/10.1016/j.ijpharm.2013.01.052 
86. Kheawfu, K., Pikulkaew, S., Rades, T., Müllertz, A., Okonogi, S. (2018). Development and characterization of clove oil nanoemulsions and self-microemulsifying drug delivery systems. Journal of Drug Delivery Science and Technology, 46, 330-338. doi: http://doi.org/10.1016/j.jddst.2018.05.028

87. Ishak, R. A. H., Osman, R. (2015). Lecithin/TPGS-based spray-dried self-microemulsifying drug delivery systems: In vitro pulmonary deposition and cytotoxicity. International Journal of Pharmaceutics, 485 (1-2), 249-260. doi: http://doi.org/10.1016/ j.ijpharm.2015.03.019

88. Vasconcelos, T., Marques, S., Sarmento, B. (2018). Measuring the emulsification dynamics and stability of self-emulsifying drug delivery systems. European Journal of Pharmaceutics and Biopharmaceutics, 123, 1-8. doi: http://doi.org/10.1016/j.ejpb.2017.11.003

89. Christiansen, M. L., Holm, R., Abrahamsson, B., Jacobsen, J., Kristensen, J., Andersen, J. R., Müllertz, A. (2016). Effect of food intake and co-administration of placebo self-nanoemulsifying drug delivery systems on the absorption of cinnarizine in healthy human volunteers. European Journal of Pharmaceutical Sciences, 84, 77-82. doi: http://doi.org/10.1016/j.ejps.2016.01.011

90. Kauss, T., Gaubert, A., Tabaran, L., Tonelli, G., Phoeung, T., Langlois, M.-H. et. al. (2018). Development of rectal selfemulsifying suspension of a moisture-labile water-soluble drug. International Journal of Pharmaceutics, 536 (1), 283-291. doi: http://doi.org/10.1016/j.ijpharm.2017.11.067

91. Kadu, P. J., Kushare, S. S., Thacker, D. D., Gattani, S. G. (2010). Enhancement of oral bioavailability of atorvastatin calcium by self-emulsifying drug delivery systems (SEDDS). Pharmaceutical Development and Technology, 16 (1), 65-74. doi: http://doi.org/10.3109/ 10837450903499333

92. Baghel, P., Roy, A., Verma, S., Satapathy, T., Bahadur, S. (2020). Amelioration of lipophilic compounds in regards to bioavailability as self-emulsifying drug delivery system (SEDDS). Future Journal of Pharmaceutical Sciences, 6 (1). doi: http://doi.org/10.1186/s43094-020-00042-0

93. Yi, T., Wan, J., Xu, H., Yang, X. (2008). A new solid self-microemulsifying formulation prepared by spray-drying to improve the oral bioavailability of poorly water soluble drugs. European Journal of Pharmaceutics and Biopharmaceutics, 70 (2), 439-444. doi: http://doi.org/10.1016/j.ejpb.2008.05.001

94. Patel, D., Sawant, K. K. (2007). Oral Bioavailability Enhancement of Acyclovir by Self-Microemulsifying Drug Delivery Systems (SMEDDS). Drug Development and Industrial Pharmacy, 33 (12), 1318-1326. doi: http://doi.org/10.1080/03639040701385527

95. Shen, H., Zhong, M. (2006). Preparation and evaluation of self-microemulsifying drug delivery systems (SMEDDS) containing atorvastatin. Journal of Pharmacy and Pharmacology, 58 (9), 1183-1191. doi: http://doi.org/10.1211/jpp.58.9.0004

96. Singh, A. K., Chaurasiya, A., Awasthi, A., Mishra, G., Asati, D., Khar, R. K., Mukherjee, R. (2009). Oral Bioavailability Enhancement of Exemestane from Self-Microemulsifying Drug Delivery System (SMEDDS). AAPS PharmSciTech, 10 (3), $906-$ 916. doi: http://doi.org/10.1208/s12249-009-9281-7

97. Truong, D. H., Tran, T. H., Ramasamy, T., Choi, J. Y., Lee, H. H., Moon, C. et. al. (2015). Development of Solid SelfEmulsifying Formulation for Improving the Oral Bioavailability of Erlotinib. AAPS PharmSciTech, 17 (2), 466-473. doi: http://doi.org/10.1208/s12249-015-0370-5

98. Xu, Y., Wang, Q., Feng, Y., Firempong, C. K., Zhu, Y., Omari-Siaw, E. et. al. (2016). Enhanced oral bioavailability of [6]Gingerol-SMEDDS: Preparation, in vitro and in vivo evaluation. Journal of Functional Foods, 27, 703-710. doi: http://doi.org/10.1016/ j.jff.2016.10.007

99. Mahmood, A., Bernkop-Schnürch, A. (2019). SEDDS: A game changing approach for the oral administration of hydrophilic macromolecular drugs. Advanced Drug Delivery Reviews, 142, 91-101. doi: http://doi.org/10.1016/j.addr.2018.07.001

100. Kazi, M., Al-Swairi, M., Ahmad, A., Raish, M., Alanazi, F. K., Badran, M. M. et. al. (2019). Evaluation of SelfNanoemulsifying Drug Delivery Systems (SNEDDS) for Poorly Water-Soluble Talinolol: Preparation, in vitro and in vivo Assessment. Frontiers in Pharmacology, 10. doi: http://doi.org/10.3389/fphar.2019.00459

101. Peltier, S., Oger, J.-M., Lagarce, F., Couet, W., Benoît, J.-P. (2006). Enhanced Oral Paclitaxel Bioavailability After Administration of Paclitaxel-Loaded Lipid Nanocapsules. Pharmaceutical Research, 23 (6), 1243-1250. doi: http://doi.org/10.1007/s11095006-0022-2

Received date 07.07.2020

Accepted date 30.07.2020

Published date 31.08.2020

Sanjib Bahadur, PhD, Associate Professor, Department of Pharmaceutics, Columbia Institute of Pharmacy, Vill. Tekari, Near Vidhan Sabha, Raipur, Chhattisgarh, India, 493111

E-mail: sanjib_pharmacist@yahoo.co.in

Kamesh Yadu, Department of Pharmaceutics, Columbia Institute of Pharmacy, Vill. Tekari, Near Vidhan Sabha, Raipur, Chhattisgarh, India, 493111

E-mail:k.yadu19962015@gmail.com

Pragya Baghel, PhD, Assistant Professor, Department of Pharmaceutics, Columbia Institute of Pharmacy, Vill. Tekari, Near Vidhan Sabha, Raipur, Chhattisgarh, India, 493111

E-mail: pragyabaghe188@gmail.com

Tripti Naurange, Department of Pharmaceutics, Columbia Institute of Pharmacy, Vill. Tekari, Near Vidhan Sabha, Raipur, Chhattisgarh, India, 493111

E-mail: triptinaurange@gmail.com

Manisha Sahu, Department of Pharmaceutics, Columbia Institute of Pharmacy, Vill. Tekari, Near Vidhan Sabha, Raipur, Chhattisgarh 493111, India

E-mail: manishasahu0411@gmail.com 